Dong, JC; Nimori, S; Kawabata, K; Goto, H, Induction of Helical Structure by Sesamin, and Production of Oriented Helical Polymer with Liquid Crystal Magneto-Electrochemical Polymerization, Journal of Polymer Science Part APolymer Chemistry, 55, 11, 1894-1899 (2017)

\title{
Induction of helical structure by sesamin, and production of oriented helical polymer with liquid crystal magneto-electrochemical polymerization
}

\author{
Jiuchao Dong1, Shigeki Nimori², Kohsuke Kawabata ${ }^{1}$, Hiromasa Goto1 \\ ${ }^{1}$ Division of Materials Science, Faculty of Pure and Applied Sciences, University of Tsukuba, \\ Tsukuba, Ibaraki 305-8573, Japan \\ ${ }^{2}$ Tsukuba Magnet Laboratory, National Institute for Materials Science, Sakura 3-13, Tsukuba, \\ Ibaraki 305-0003, Japan \\ Correspondence to: Hiromasa Goto (E-mail: gotoh@ims.tsukuba.ac.jp)
}

\begin{abstract}
Sesamin was employed as a chiral dopant for preparing cholesteric liquid crystals with righthanded helical architecture. Helical twisting power of sesamin is to be $13.4 \mu^{-1}$. Electrochemical polymerizations were carried out with sesamin-induced cholesteric liquid crystal electrolyte solution for obtaining conjugated polymer films with helical structure. The film was transcribed the helical order from the liquid crystal electrolyte solution with helical structure produced by sesamin during the polymerization process. The helical axes of the macromolecular superstructure of the polymer films were oriented in a magnetic field of $4.5 \mathrm{~T}$. This results demonstrated liquid crystal magneto-electrochemical polymerization with helical structure induced by sesamin as a natural chiral compound.
\end{abstract}

KEYWORDS: sesamin, chiral dopant, helical superstructure, conjugated polymer, electrochemical polymerization

\section{INTRODUCTION}

Chiral dopant is a material that can induce a formation of chiral liquid crystal phase from achiral liquid crystals (LCs). After addition of chiral dopants to achiral LC, the initial liquid crystal phase can transform to a chiral liquid crystal phase with helical molecular superstructure. ${ }^{1-4}$ Even though chiral LCs can also be obtained by introducing chirality centers into liquid crystal molecules directly, ${ }^{5}$ the helical pitches of synthesized chiral LC by this method are difficult to predict and adjust. While, employment of chiral dopants can easily tune helical pitch by changing amount of addition in the nematic LC. Therefore, this is more favorable for application in chiral liquid crystals for LC displays, especially in the development of blue-phase LC technology. ${ }^{6-8}$

Many chiral dopants have been synthesized. Some compounds showed very high helical twisting power with excellent solubility or tunable helical twisting power. ${ }^{9-13}$ However, synthesis of these 
Dong, JC; Nimori, S; Kawabata, K; Goto, H, Induction of Helical Structure by Sesamin, and Production of Oriented Helical Polymer with Liquid Crystal Magneto-Electrochemical Polymerization, Journal of Polymer Science Part APolymer Chemistry, 55, 11, 1894-1899 (2017)

chiral dopants is usually complicated and costly. Therefore, our group focuses on natural chiral compounds. Many natural compounds have asymmetric centers, like vitamins, borneol, glucose, and menthol, some of which show potential as chiral dopants. ${ }^{14}$

Sesamin is a kind of lignin isolated from the bark of fagara plants and sesame oil. This natural compound has some attractive properties for medicines, such as reducing cholesterol absorption, anti-carcinogenesis effect, anti-hypertension effect, lipid-lowering effect and improving liver function, thus attracting considerable attention as healthy diet components. ${ }^{15-17}$ Sesamin is a chiral compound with four asymmetric centers and a $\mathrm{C} 2$ symmetry axis. These properties allow sesamin to work as a chiral dopant (chiral inducer) for production of cholesteric LC (or N*-LC) with helical aggregation super structure from nematic LC having no helical structure.

Our group has developed a new electrochemical polymerization for fabricating conjugated polymer films. This new method can be used for construction of helical superstructure molecular alignment by employing cholesteric LCs as the reaction media. ${ }^{18-20}$ Cholesteric LC is a chiral liquid crystal and has helical molecular arrangement. Polymerization in cholesteric LC allows production of polymers with optical activity originating from helical molecular arrangement. Moreover, the films show macroscopic periodic concave-convex surface structure, which functions as a diffraction grating. Due to these features, the films can be endowed with various functionalities, such as circular dichroism, optical absorption, selective reflection, decreasing surface reflection, changing friction coefficient or adjusting contact angle.

In this research, chiral dopant (or chiral inducer) properties of sesamin, helical twisting power and induction of helicity, are evaluated. Sesamin is used to prepare cholesteric liquid crystal, which is employed for electrochemical polymerization of conjugated polymers. Optical properties of the resultant conjugated polymer film obtained in the cholesteric liquid crystal were examined by the UV-vis absorption and circular dichroism (CD) spectroscopy measurements. Moreover, electrochemical polymerization is also carried out under magnetic field. Magnetic field can function for molecular alignment of cholesteric LC molecules. Magnetic field allows tuning of helical superstructure of resultant conjugated polymer films during the polymerization through tuning of helicity of matrix LC (cholesteric LC). Optical texture of the polymer films obtained under external magnetic field are observed by polarizing optical microscopy (POM).

\section{EXPERIMENTAL}

\section{Electrochemical polymerization}

Chiral dopant sesamin ( $3 \mathrm{~mol} \%$ ), supporting salt tetrabutylammonium perchlorate (TBAP) (0.5 mol\%) and monomer 3,7-di(2-thienyl)dibenzothiophene (DTbt) $(0.3 \mathrm{~mol} \%)$ were dissolved in nematic LC 4-cyano-4'-hexylbiphenyl (6CB) with sufficient mix for preparing cholesteric LC electrolyte solution. This electrolyte solution was injected into a reaction cell consisting of two indium-tin-oxide (ITO)-coated glass electrodes and a Teflon spacer (thickness $0.20 \mathrm{~mm}$ ). The LC was warmed up to form isotropic phase. Subsequent natural cooling to room temperature allows to proceed phase transition of the electrolyte solution to cholesteric phase from isotropic. Formation of non-oriented cholesteric LC phase in the cell was confirmed by the POM observation. Then, polymerization was carried out with application of direct current $(4.0 \mathrm{~V})$ at room temperature. After $20 \mathrm{~min}$, a resultant polymer film (P-DTbt) was obtained, and washed 
with dichloromethane (DCM). Chemical structures of constituents of the electrolyte solution are shown in Scheme 1.

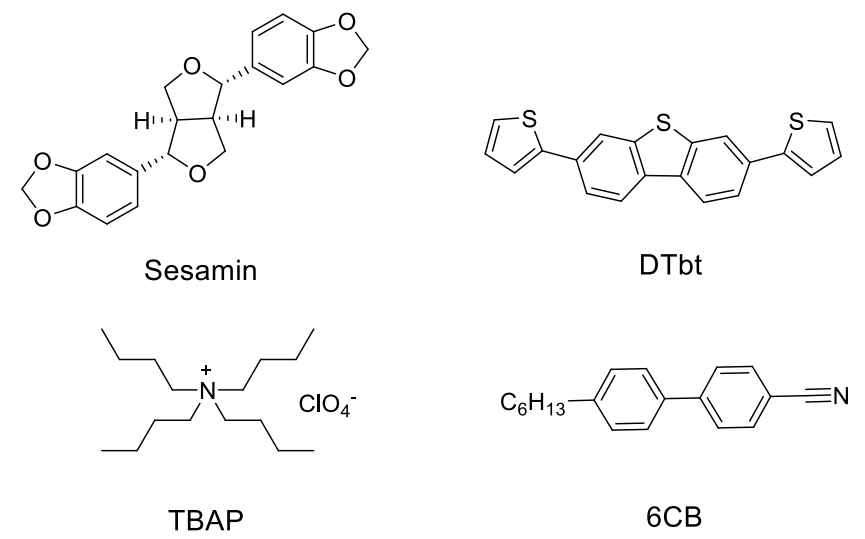

Scheme 1. Chemical structure of sesamin, DTbt, TBAP and 6CB.

\section{Chemicals and characterization methods}

Sesamin and TBAP were purchased from Tokyo Chemical Industry (TCI, Japan). Synthesis of 2,7-di(2-furyl)-fluorene have been previously reported. ${ }^{19}$ 6CB (nematic LC) was purchased from Merck.

Microscopic observations were performed with crossed Nicols using a Nikon Eclipse LV100 (Japan) polarizing optical microscope (POM) equipped with a TH-600PM and L-600 heating and cooling stage (Linkam, UK). UV-vis absorption spectra were measured with a JASCO V-630 spectrophotometer. Circular dichroism (CD) optical absorption spectroscopy measurements were carried out with a JASCO J-720 spectrometer and an ORDE-307W ORD unit (Japan).

\section{RESULTS AND DISCUSSION}

\section{Helical twisting power and helical sense}

Grandjean-Cano wedge method and miscibility test method estimate helical twisting power and helical sense. ${ }^{21}$ Sesamin was dissolved in 6CB (3 mol\% vs. 6CB). The blend LC showed a fanlike texture at room temperature (Figure 1), which is a typical optical texture of short-pitch cholesteric LC, indicating the sesamin successfully induced cholesteric phase in 6CB. A POM image of the LC in the Grandjean-Cano cell is shown in Figure 2. Helical twisting power $\left(\beta_{\mathrm{M}}\right)$ can be calculated as

$$
\beta_{\mathrm{M}}=(p c)^{-1}
$$

Here, $p$ is helical pitch of the mixture, and $c$ is concentration (in mol\%) of the chiral dopant. Grandjean-Cano wedge method revealed that helical pitch of this blend LC is to be $2.49 \mu \mathrm{m} . \beta_{\mathrm{M}}$ is to be $13.4 \mu \mathrm{m}^{-1}$. 
Cholesteryl oleyl carbonate was employed as a reference for definition of helical sense of sesamin in the miscibility test. This compound can induce left-handed helical architecture in 6CB. We prepared a standard cholesteric LC with cholesteryl oleyl carbonate and 6CB. Precise adjustment of the concentration of sesamin in 6CB to prepare this standard LC with the same helical pitch as the standard LC. Figure 3 shows the POM image in the miscibility test at room temperature. The sample was placed in the left region in the glass cell, and the standard LC was placed in the right region. Then, an obvious boundary between two LCs appeared at the center region. Both LC show fingerprint textures. However, fingerprint textures unwind at the boundary to show nematic phase with no helical structure, indicating the sesamin has opposite helical sense against cholesteryl oleyl carbonate. This result indicates that sesamin induces right-handed helical architecture in 6CB.

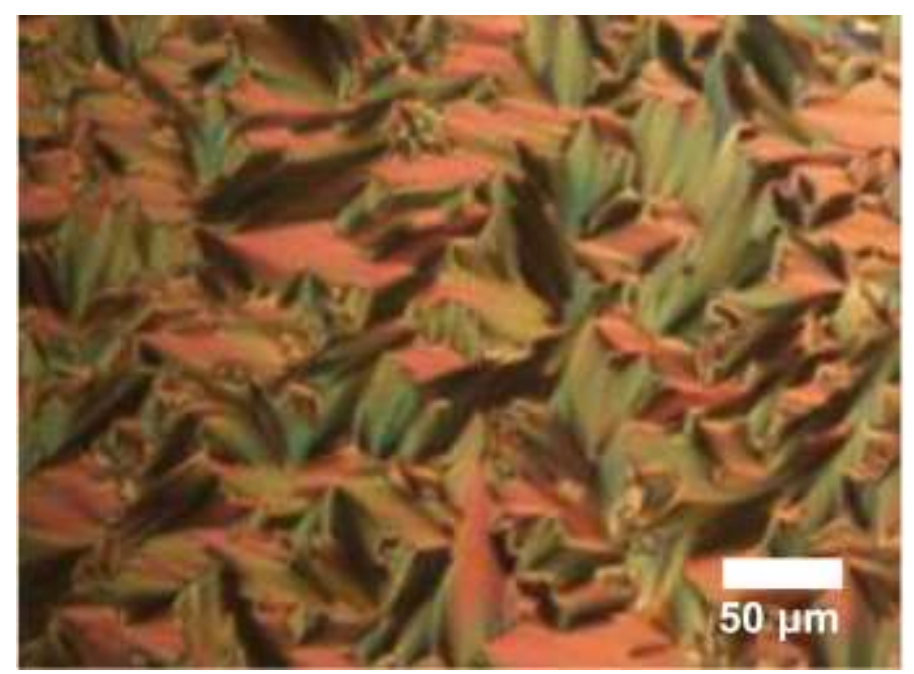

Figure 1. Polarizing optical microscopy (POM) image of blend cholesteric LC (sesamin $3 \mathrm{~mol} \%$ in $6 \mathrm{CB})$.

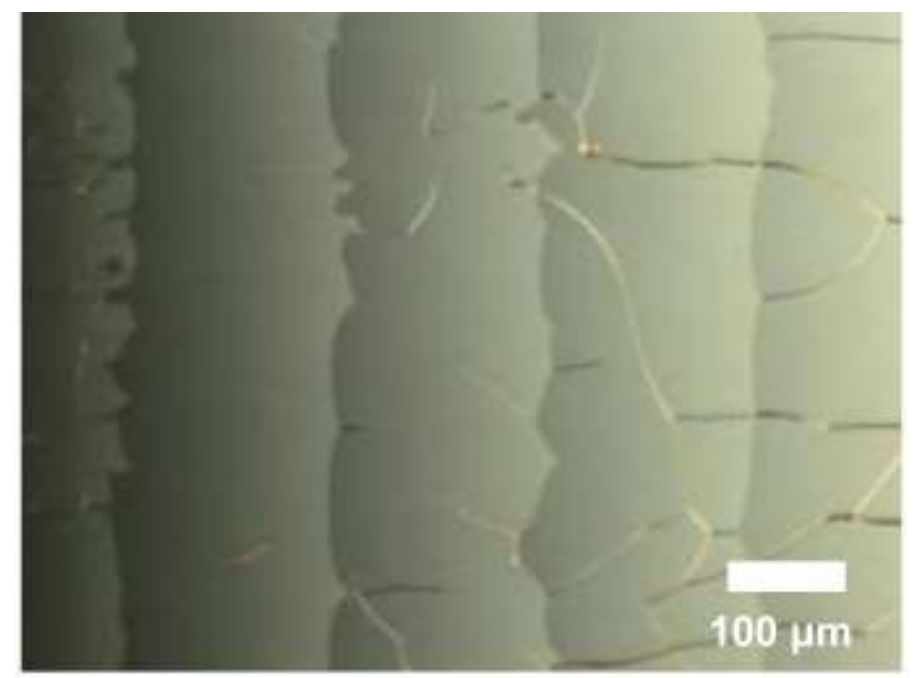

Figure 2. POM image of the blend LC sample in Grandjean-Cano cell. (sesamin $3 \mathrm{~mol} \%$ in $6 \mathrm{CB}$ ). 


\section{Electrochemical polymerization in sesamin-induced cholesteric LC}

The LC solution containing sesamin was employed for electrochemical polymerization of monomer DTbt. Optical texture of the resultant P-DTbt film was observed by the POM (Figure 4), revealing alternating dark and bright streaks pattern, which is similar to the fingerprint texture of cholesteric LC. We obtained the UV-vis optical absorption and the CD spectra (Figure 5). The polymer shows an optical absorption at $300-550 \mathrm{~nm}$ due to $\pi-\pi^{*}$ transition with maximum absorption wavelength at $400 \mathrm{~nm}$. Meanwhile, the CD spectra show a positive absorption at 420$580 \mathrm{~nm}$. The maximum absorption wavelength of the CD spectra appears at $487 \mathrm{~nm}$. A weak negative CD signal can be observed at short wavelengths.

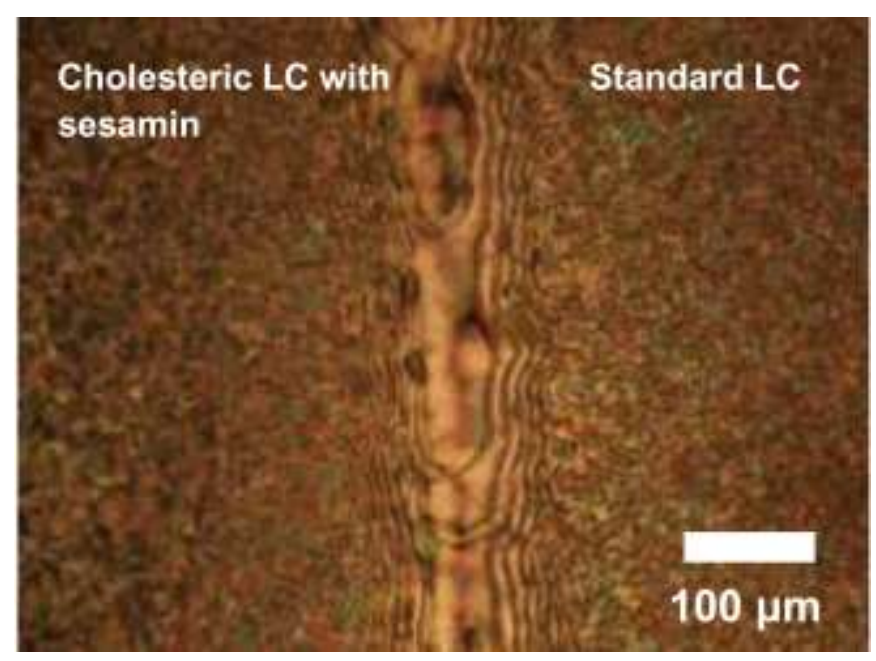

Figure 3. POM image of miscibility test.

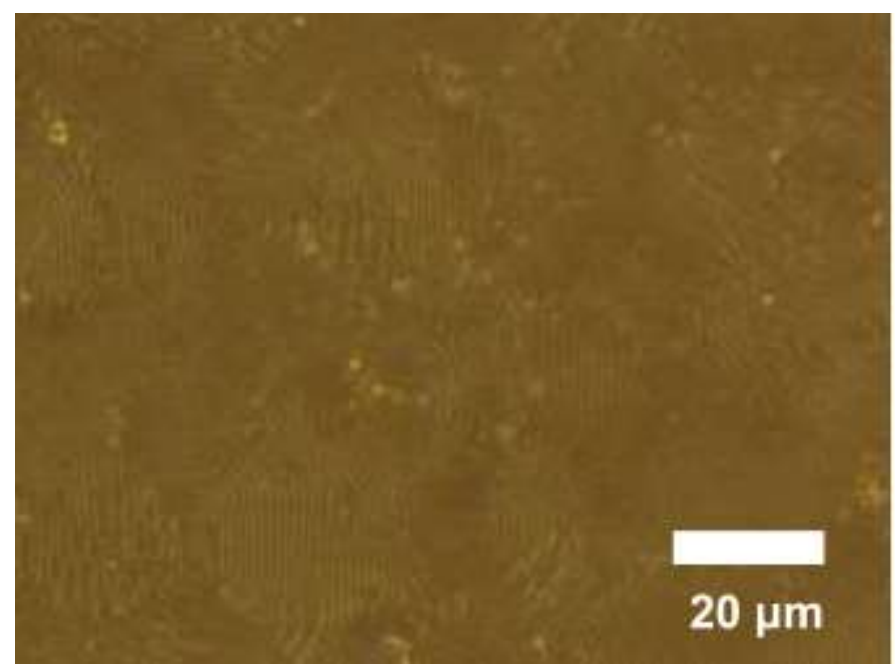

Figure 4. POM image of P-DTbt film obtained with electrochemical polymerization in sesamin induced cholesteric LC. 
The negative signal appears after the positive signal from long wavelengths. This is referred to as positive Cotton effect. ${ }^{22-24}$ The Cotton effect can be employed to determine helical direction of optically active samples. This positive Cotton effect indicates that the chromophores arrange in right-handed helical order. The zero cross position from positive to negative signal in the $\mathrm{CD}$ is located at around the maximum absorption wavelength in the UV-vis. This phenomenon can be attributed to Davydov splitting, which indicates that two adjacent chromophores are close enough to have strong interaction, and their energy levels are split. In general, this splitting in the UV-vis is difficult to observe for aggregated conjugated polymers, because the two split absorption bands almost overlap in one signal.

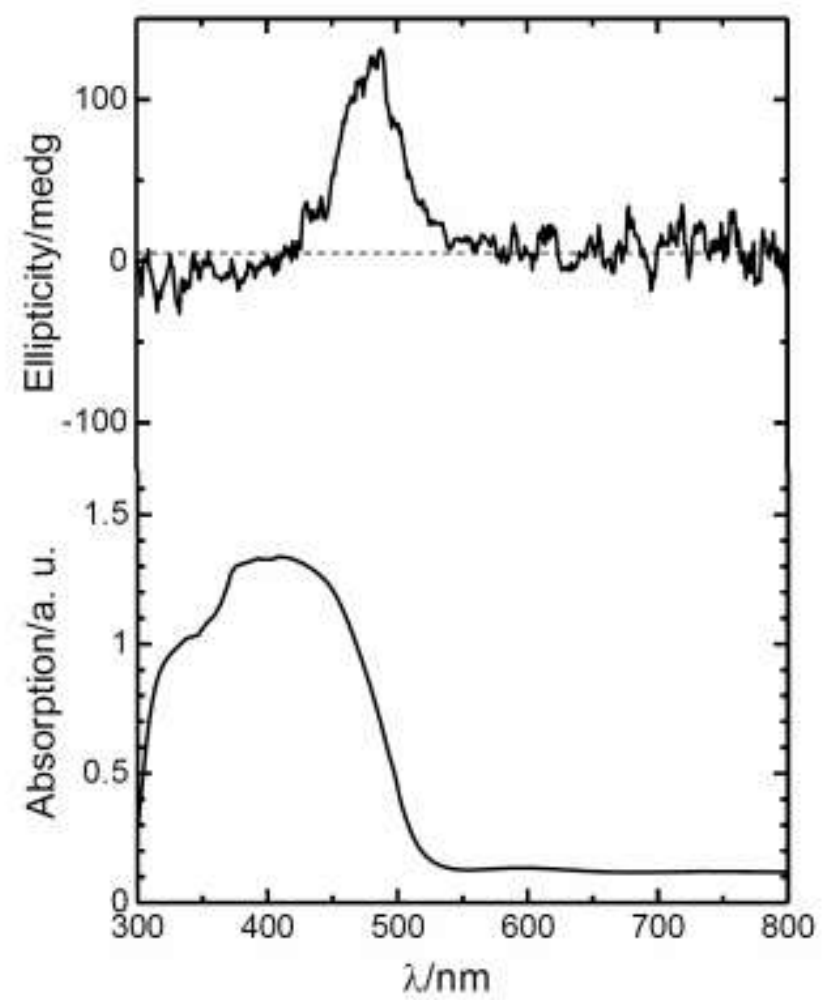

Figure 5. CD spectra (top) and UV-vis opticl absorption spectra (bottom) of P-DTbt film.

This result indicates that P-DTbt forms right-handed helical order in the aggregation. In particular, this helical superstructure has the same helical order as sesamin-induced cholesteric LC. Figure 6 displays possible helical superstructure of the cholesteric LC and P-DTbt. P-DTbt transcribes the molecular orientation order from the blend LC in the electrochemical polymerization process. Therefore, the polymer film shows a similar optical texture with cholesteric LC in POM observation. 


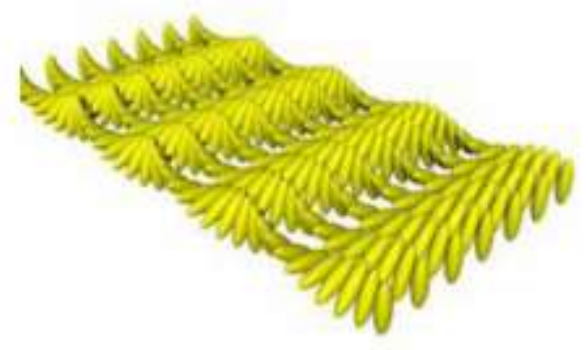

Cholesteric LC electrolyte solution

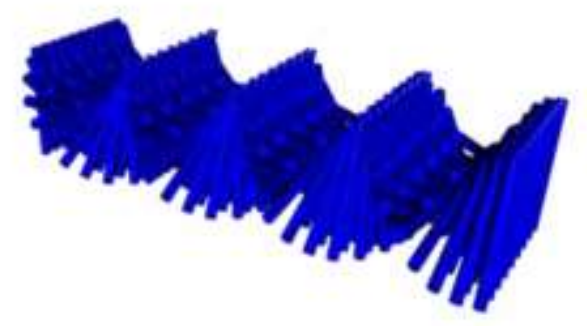

Polymer film

Figure 6. Possible helical aggregation of the cholesteric liquid crystal electrolyte solution and resultant polymer film.

\section{Superstructure adjustment by magnetic field}

Direction of helical axes in the entire film is random in this polymerization. To control molecular orientation, polymerization in magnetic field was carried out. Since the helical superstructure of P-DTbt films transcribes the molecular order of cholesteric LC, molecular order of the resultant polymer can be tuned through orientation of the matrix LC. LC molecules usually show anisotropic magnetic susceptibility, which allows LC molecules to align uniaxial direction along the magnetic field. ${ }^{25}$ LC molecules can be oriented completely with very strong magnetic field. However, the intense magnetic field rewind helical structure of the cholesteric phase, resulting change to nematic phase with no helical structure. An appropriate strength of the magnetic field for cholesteric LC can afford to obtain uniaxial cholesteric LC orientation with helical structure (Figure 7). 


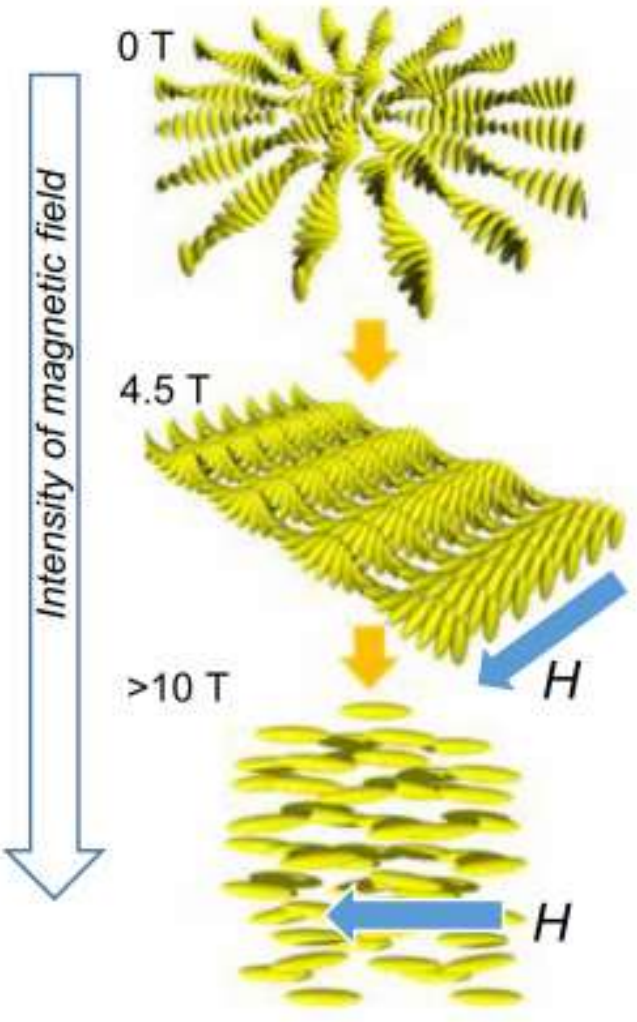

Figure 7. Alignment of cholesteric liquid crystal under magnetic fields.

Electrochemical polymerizations were carried out at $0.6,1.0,1.4,2.0,2.5,3.0,3.5,4.0,4.5,6.0$, 8.0, 10 and $12 \mathrm{~T}$. The polymer films thus obtained in the magnetic fields are shown in Figs. 8-10, where gradual change of optical texture can be observed. At $0.6 \mathrm{~T}$, the streaks form circular rings. There is no obvious difference between the polymer films obtained under zero magnetic field (Figure 4) and 0.6 T. Curvature of the streaks decrease at $1.0 \mathrm{~T}$, resulting the circular rings was elongated. Almost all curves become straight lines at $4.0 \mathrm{~T}$, where no ellipses are observed. The polymer obtained at $4.5 \mathrm{~T}$ shows quasi-complete orientation of helical axes. 
Dong, JC; Nimori, S; Kawabata, K; Goto, H, Induction of Helical Structure by Sesamin, and Production of Oriented Helical Polymer with Liquid Crystal Magneto-Electrochemical Polymerization, Journal of Polymer Science Part APolymer Chemistry, 55, 11, 1894-1899 (2017)

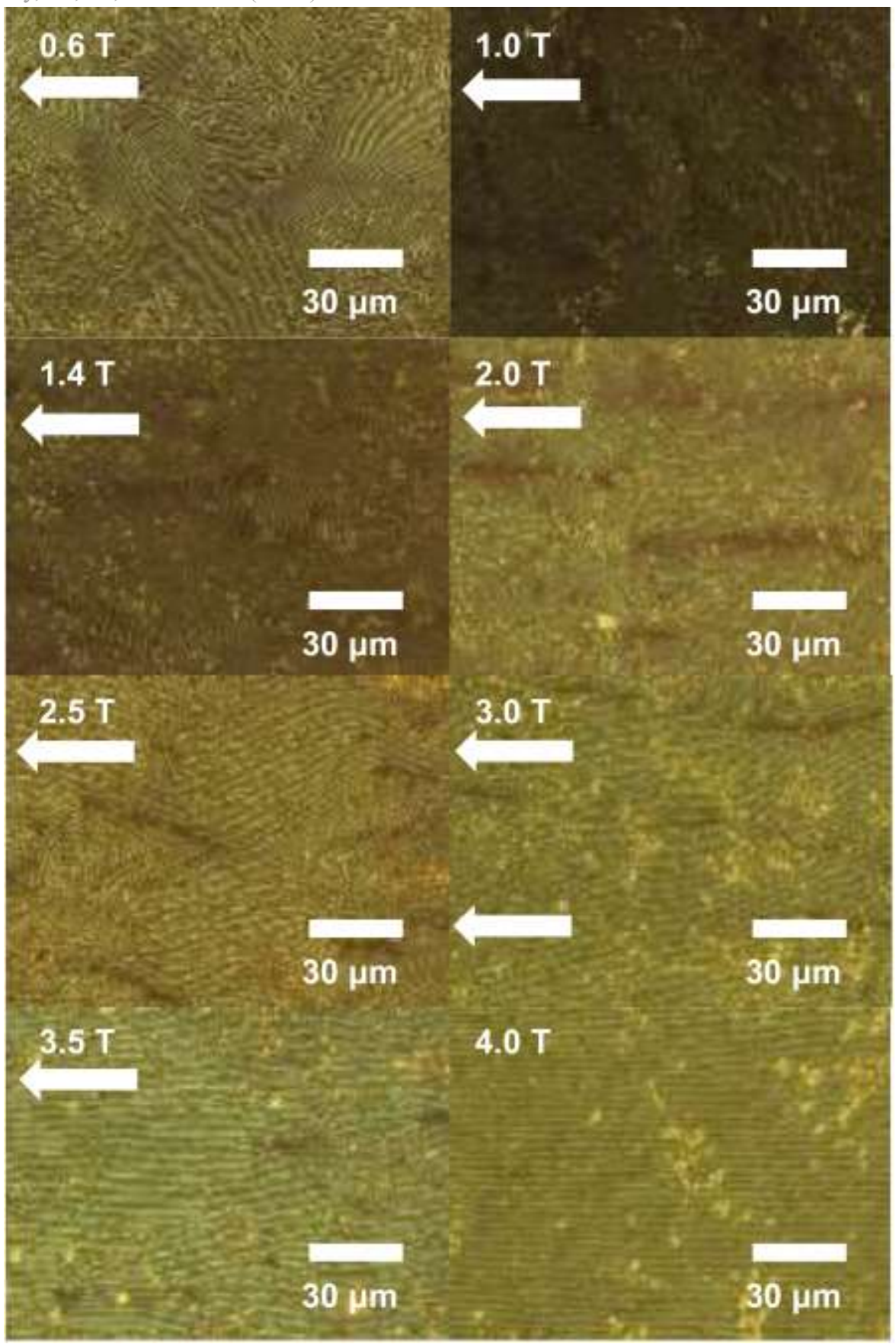

Figure 8. POM images of P-DTbt films prepared by electrochemical polymerization under magnetic field of 0.6-4.0 T. Arrows indicate direction of magnetic field. 


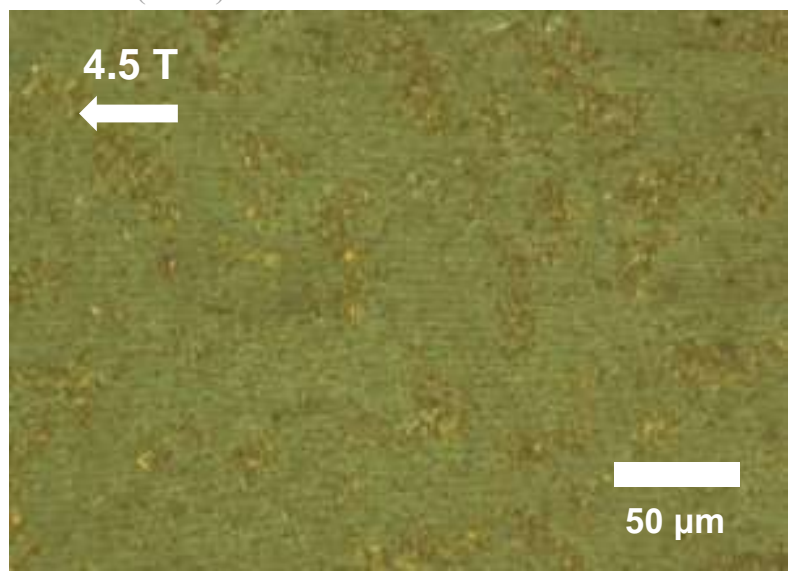

Figure 9. POM image of P-DTbt film obtained by electrochemical polymerization under 4.5 T.

The streaks gradually disappear at $6.0 \mathrm{~T}$ and $8.0 \mathrm{~T}$, and the streaks completely disappear at $10 \mathrm{~T}$ and $12 \mathrm{~T}$, indicating helical structure of the polymer completely unwinded. The intense magnetic field induced nematic LC via unwind of helical structure of cholesteric LC. Simultaneously, the nematic LC aligns one-direction along the magnetic field. Polymerization in the oriented LC allows production of the excellent uniaxial oriented film.

Polymerization in the LCs under the magnetic field of $4.5 \mathrm{~T}$ produces helical periodicity uniaxially aligned polymer, and $\geq 10 \mathrm{~T}$ uniaxially aligned polymer.

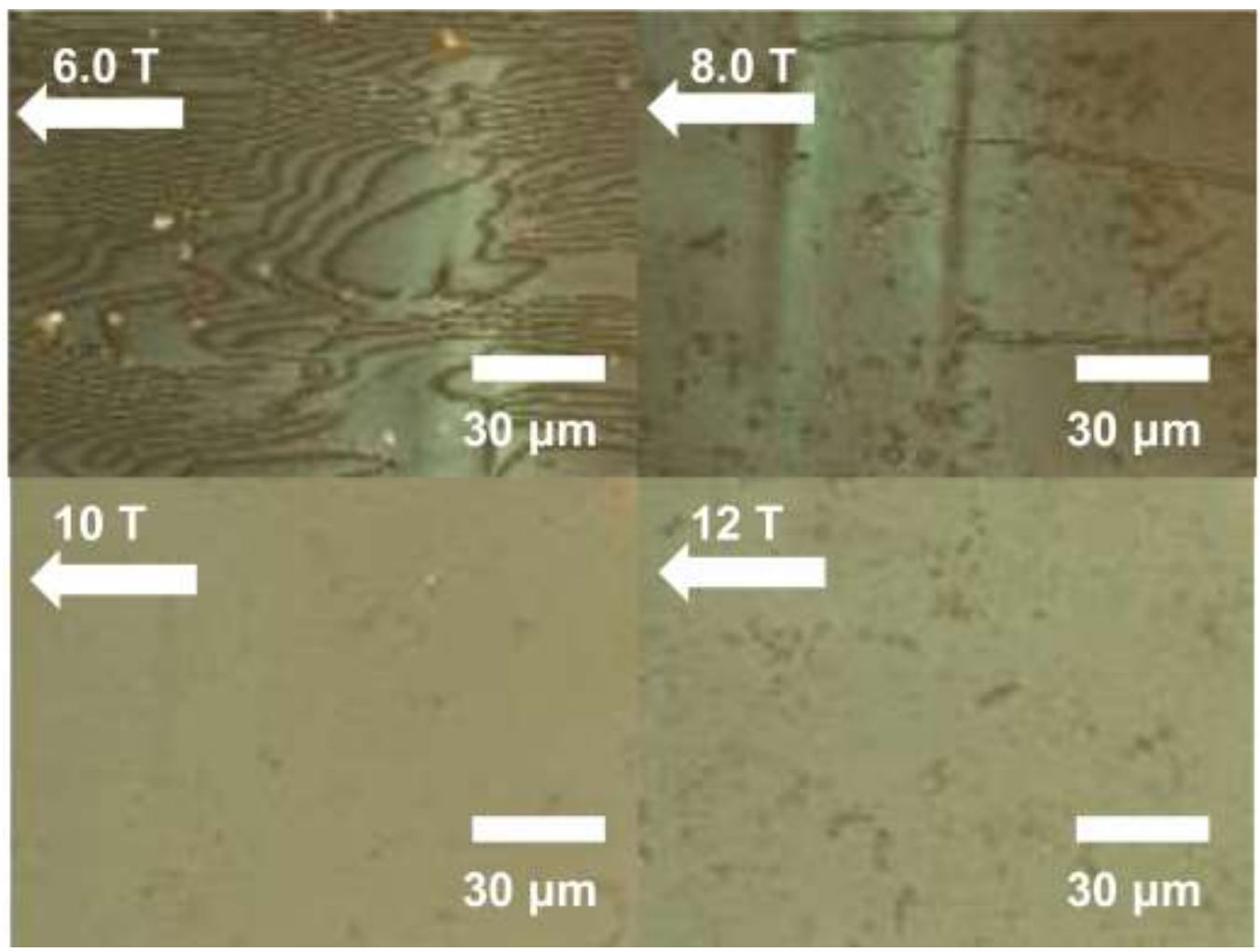

Figure 10. POM images of P-DTbt films obtained by electrochemical polymerization with magnetic field orientation (6.0-12 T). 


\section{CONCLUSIONS}

We demonstrated the possibility of sesamin as a chiral induction agent to construct cholesteric LCs. This natural compound has good affinity with synthetic LC, and can induce right-handed helical architecture.

The electrochemical prepared polymer in the sesamin-induced cholesteric LC having righthanded helical superstructure, which is consistent with helical direction of matrix cholesteric LC electrolyte solution, indicating that the polymer film transcribes the molecular order of the LC during the polymerization. We found appropriate intensity of magnetic field to align the helical superstructure of LC molecules and resultant polymer films. The helical axes can be uniaxially oriented under $4.5 \mathrm{~T}$.

\section{ACKNOWLEDGEMENTS}

We would like to thank the National Institute for Materials Science for the use of the $12 \mathrm{~T}$ magnetic field device. We would like to thank Aohan Wang for her help and support.

\section{REFERENCES AND NOTES}

1. K. Kakisaka, H. Higuchi, Y. Okumura, H. Kikuchi, Chem. Lett., 2014, 43, $624-625$.

2. N. L. Babak, O. V. Shishkin, S. V. Shishkina, I. M. Gella, V. I. Musatov, N. B. Novikova, V. V. Lipson, Struct. Chem., 2015, $1-9$.

3. J. A. Rego, J. A.A. Harvey, A. L. MacKinnon, E.Gatdula, Liq. Cryst., 2010, 37, 37 - 43

4. M. P. Thompson, R. P. Lemieux, J. Mater. Chem., 2007, 17, 5068 - 5076.

5. G-Y. Yeap, S. Balamurugan, M. V. Srinivasan, P. Kannan, New J. Chem., 2013, 37, 1906 1911.

6. L. Rao, J. Yan, S-T. Wu, Journal of the SID, 2010, 18, $954-959$.

7. K. Kakisaka, H. Higuchi, Y. Okumura, H. Kikuchi, J. Mater. Chem. C., 2014, 2, 6467 - 6470.

8. N. Li, Z. Li, X. Zhang, R. Hua, Int. J. Mol. Sci., 2013, 14, 23257 - 23273.

9. K. Kishikawa, T. Sugiyama, T. Watanabe, S. Aoyagi, M. Kohri, T. Taniguchi, M. Takahashi, S. Kohmoto, J. Phys. Chem. B, 2014, 118, $10319-10332$.

10. Y-L. Wu, F. Ferroni, S. Pieraccini, W. B. Schweizer, B. B. Frank, G. P. Spadab, F. Diederich, Org. Biomol. Chem., 2012, 10, $8016-8026$.

11. Y. Li, Q. Li, Org. Lett., 2012, 14, $4362-4365$.

12. J. Yoshida, G. Watanabe, K. Kakizawa, Y. Kawabata, Hf. Yuge, Inorg. Chem., 2013, 52, $11042-11050$.

13. Y. Kim, N. Tamaoki, J. Mater. Chem. C, 2014, 2, 9258 - 9264.

14. P. Budowski, J. Am. Oil Chem.Soc, 1964, 41, $280-285$.

15. A. Matsumura, Y. Fan, H. Goto, Synth. Me., 2015, 202, 157 - 164.

16. D. Nakano, C-J. Kwak, K. Fujii, K. Ikemura, A. Satake, M. Ohkita, M. Takaoka, Y. Ono, M. Nakai, N. Tomimori, Y. Kiso, Y. Matsumura, J PHARMACOL EXP THER, 2006, 318, 328 335.

17. T. Ide, M. Kushiro, Y. Takahashi, K. Shinohara, N. Fukuda, S. S-Yasumoto, JARQ, 2003, 37, $151-158$.

18. J. Dong, K. Kawabata, H. Goto, J. Mater. Chem. C, 2015, 3, 2024 - 2032.

19. K. Kawabata, M. Takeguchi, H. Goto, Macromolecules, 2013, 46, 2078 - 2091.

20. H. Goto, Macromolecules, 2007, 40, 1377 - 1385. 
21. H. Hayashi, A. Wang, K. Kawabata, H. Goto, Mater. Chem. Phys., 2013, 127, 816 - 824.

22. T. Yamamoto, T. Adachi, M. Suginome, ACS Macro Lett., 2013, 2, 790 - 793.

23. K. Kishikawa, T. Sugiyama, T. Watanabe, S. Aoyagi, M. Kohri, T. Taniguchi, M. Takahashi, S. Kohmoto, J. Phys. Chem. B, 2014, 118, $10319-10332$.

24. Y. Nagata, T. Nishikawa, M. Suginome, Chem. Commun., 2012, 48, 11193 - 11195.

25. K. Kawabata, S. Nimori, H. Goto, ACS Macro Lett., 2013, 2, 587 - 591.

\section{GRAPHICAL ABSTRACT}

Author name: Jiuchao Dong, Shigeki Nimori, Kohsuke Kawabata, Hiromasa Goto

Title: Induction of helical structure by sesamin, and production of oriented helical polymer with liquid crystal magneto-electrochemical polymerization

Text: Liquid crystal magneto-electrochemical polymerization induced by sesamin produces uniaxially oriented helical structure.

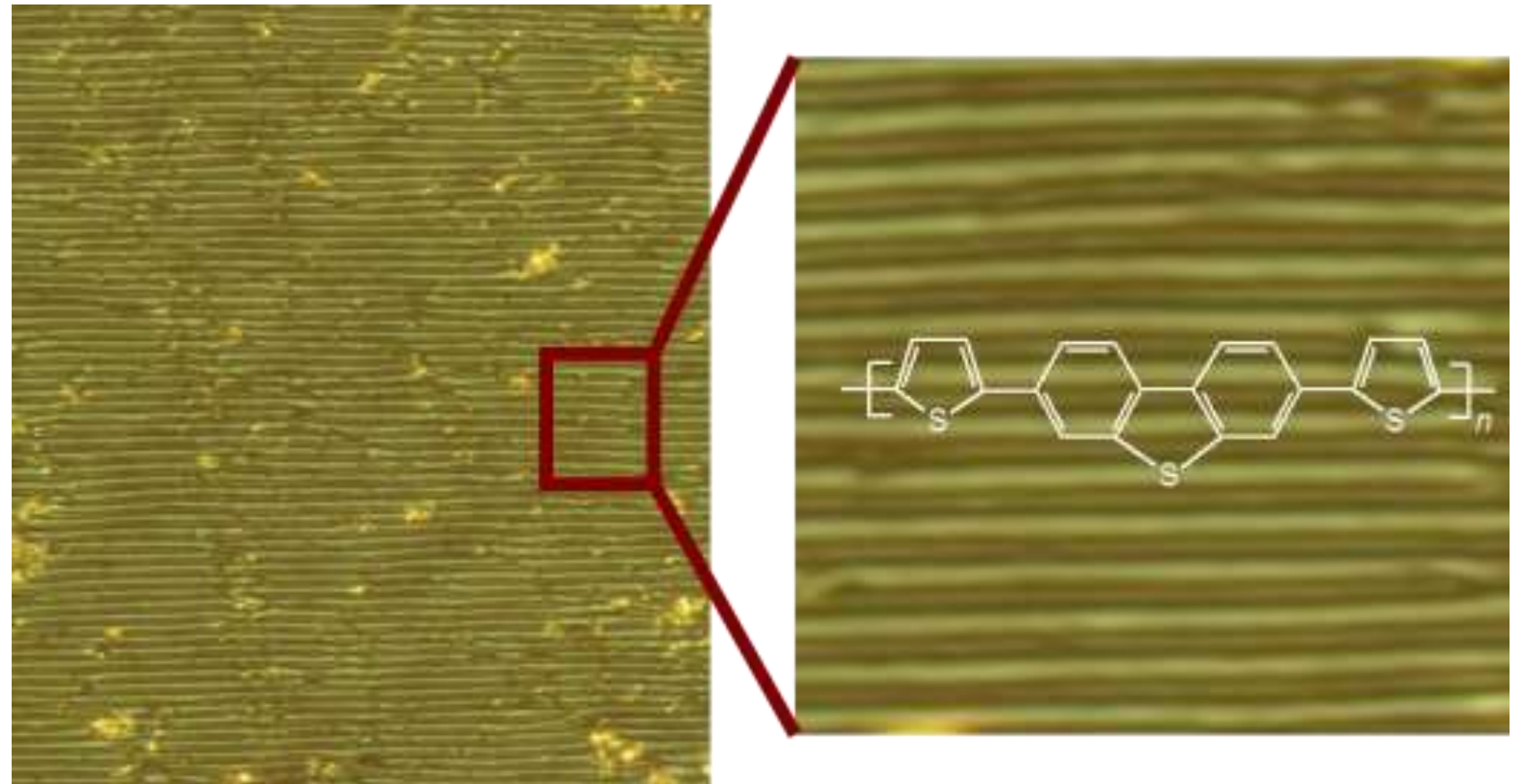


Supporting information for:

\title{
Induction of helical structure by sesamin, and production of oriented helical polymer with liquid crystal magneto- electrochemical polymerization
}

\author{
Jiuchao Dong ${ }^{1}$, Shigeki Nimori ${ }^{2}$, Kohsuke Kawabata ${ }^{1}$, Hiromasa Goto ${ }^{1, *}$
}

${ }^{1}$ Division of Materials Science, Faculty of Pure and Applied Sciences, University of Tsukuba, Tsukuba, Ibaraki 305-8573, Japan

${ }^{2}$ Tsukuba Magnet Laboratory, National Institute for Materials Science, Sen-gen, Tsukuba1-2-1, Ibaraki 305-0047, Japan

Correspondence to H. Goto, Tel: +81-298-53-5128, Fax: +81-298-53-4490

Email: gotoh@ims.tsukuba.ac.jp 


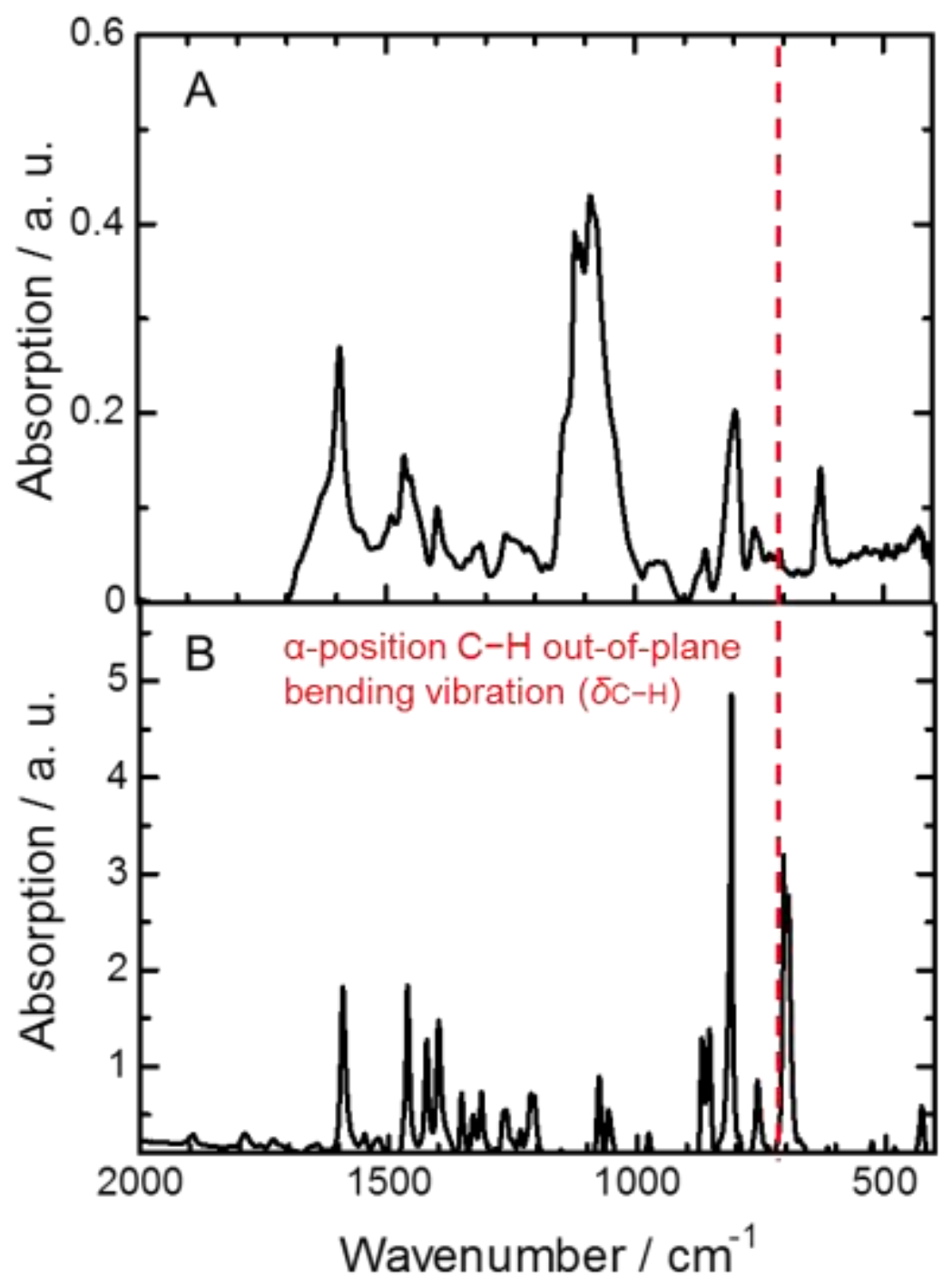

Figure S1. IR spectra of the P-DTbt film (A) and the monomer DTbt (B). Absorption band due to $\alpha$-position $\mathrm{C}-\mathrm{H}$ out-of-plane bending vibration $\left(\delta_{\mathrm{C}-\mathrm{H}}\right)$ of monomer DTbt does not appear in the IR spectrum of P-DTbt film. This elucidates that the polymers was obtained and grew via coupling between the $\alpha$-position of the monomers. 


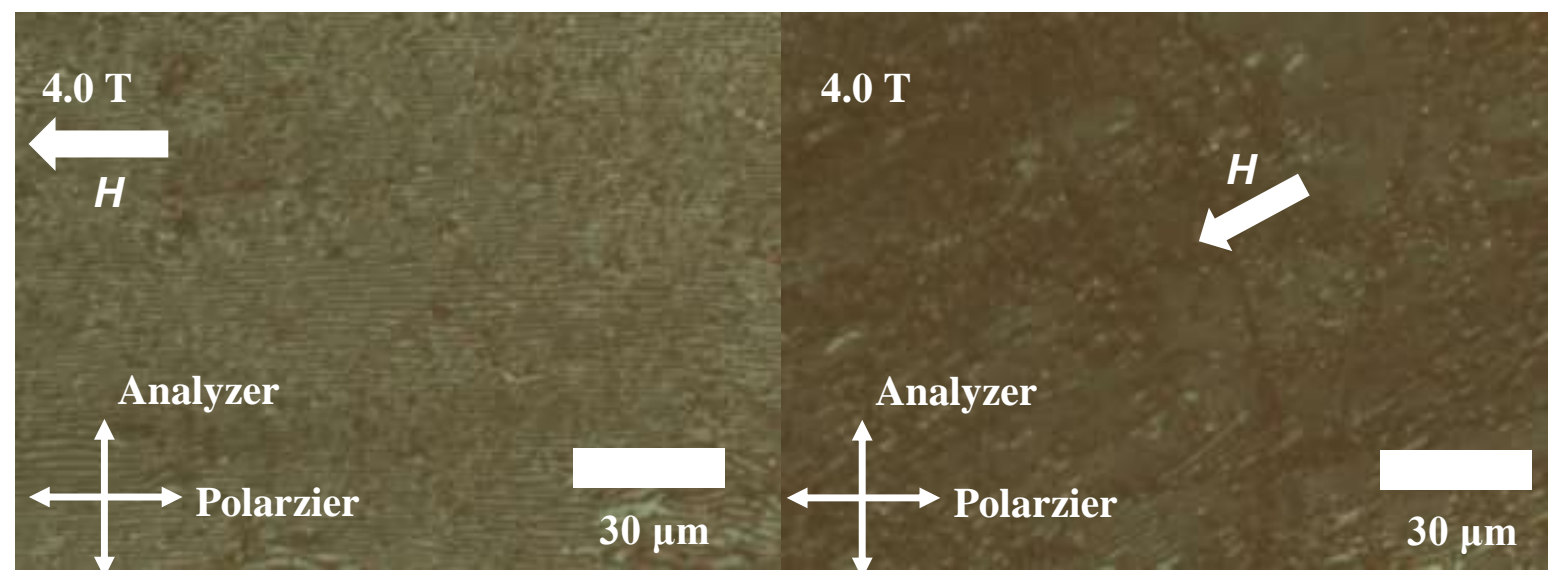

Figure S2. (Left): POM images of P-DTbt film obtained by electrochemical polymerization under magnetic field (white arrows indicate the field orientation). (Right): Magnetic field vs. polarizer $=\mathrm{ca} .30^{\circ}$ under cross nicol condition. 